\title{
Magnetic fields in the early Universe
}

\author{
Eduardo Battaner ${ }^{1,2}$ and Estrella Florido ${ }^{1,2}$ \\ ${ }^{1}$ Departamento de Física Teórica y del Cosmos, Universidad de Granada \\ Avenida Fuentenueva s/n, 18071 Granada \\ ${ }^{2}$ Institute Carlos I for Theoretical and Computational Physics. Universidad de Granada \\ email: battaner@ugr.es,estrella@ugr.es
}

\begin{abstract}
There is increasing evidence that intense magnetic fields exist at large redshifts. They could arise after galaxy formation or in very early processes, such as inflation or cosmological phase transitions, or both. Early co-moving magnetic strengths in the range 1-10 nG could be present at recombination. The possibilities to detect them in future CMB experiments are discussed, mainly considering their impact in the anisotropy spectra as a result of Faraday rotation and Alfven waves. Magnetic fields this magnitude could also have a non-negligible influence in determining the filamentary large scale structure of the Universe.
\end{abstract}

Keywords. Magnetic fields - cosmology - cosmic microwave background - large-scale structure of universe

\section{Introduction}

There are previous excellent reviews on this topic. For elaborating this one the authors have found particularly instructive those by Giovannini 2002, Giovannini 2004, Giovannini 2006 and Grasso \& Rubinstein (2001).

We could advance that we have no measurement at all of early universe magnetic fields, therefore, the topic is highly speculative. Nevertheless, there is a large amount of theoretical work, even at an embryonic state due to the lack of observational parallel work. There is also the reasonable hope that measurements will become available in the next decade. If early magnetic fields really exist our conception of the Universe should be modified and the number of parameters defining it should be enriched.

In fact, the higher redshift at which we have a reliable rotation measure is $\mathrm{z} \approx 2$. This is the case of 3C191 with an absorption line spectrum due to a wind-driven shell of gas. Rotation measures higher than $200 \mathrm{rad} / \mathrm{m}^{2}$ corresponding to field strengths in the range 0.4-4 $\mu \mathrm{G}$ (Kronberg 2005, Kronberg, Bernet, Miniati et al. 2008, this book) were detected. Radioastronomical observations of point sources limit our possibilities to Lyman- $\alpha$ forests or absorbers in front of quasars. See also Bernet, Miniati, Lilly et al. (2008). Beck (2005; this book) have comprehensive reviewed the measurements of galactic and extragalactic fields.

The present matter density is very inhomogeneous and magnetic fields should be as well inhomogeneously distributed. In particular, no measurements of magnetic fields in large scale voids have been obtained (and this would be out of present observational capabilities).

In order to observe and respect the Cosmological Principle of isotropy, we could disregard a homogeneous magnetic field within the present Hubble radius. To be specific about, we would assume $\langle\vec{B}\rangle=0$ even if $\left\langle B^{2}\right\rangle \neq 0$, i.e. the mean magnetic energy density is not vanishing. The magnetogenesis mechanism, whatever it was, could produce fields this scale, but this is unlike. Upper limits exist to disregard an ordered field on the scale of the observable universe (e.g. Widrow 2002). 
Because of the solenoidal character of the field, their distribution in space would consist in closed loops of different sizes, being their topological and array properties uncertain.

It is not surprising the fact that so many theoretical work has been developed about magnetic fields in the early universe, despite the absolute lack of measurements. There are basically three reasons: the first one is more or less sentimental: if we find magnetic fields in all astrophysical subsystems (planets, stars and interplanetary, interstellar and intergalactic media) why should a cosmological field be absent? Second, several forthcoming experiments could provide an observational detection, particularly PLANCK, but also the SKA (Beck 2008) QUIJOTE (Rubiño-Martin et al. 2008) and others. A Planck Project Constraints on Primordial Magnetic Fields is now running within the Planck Working Group on Non-Gaussianity (e.g. Battaner \& Rubiño-Martin 2008). There is a Planck Project devoted to this study. The third is that the introduction of magnetic fields in the interpretation of $\mathrm{CMB}$ and the large scale structure could modify the actual values of the parameters defining our universe, what is of higher and exciting cosmological interest.

An interesting argument was raised by Fermi. Taking into account that in a perfectly conducting fluid, magnetic fields were long lived, and considering the isotropy of the cosmic rays, he was able to obtain an order of magnitude of the galactic magnetic field. He then considered the possibility of a primordial origin of this field. Cosmic rays are confined into the galaxy if their Larmor radius is lower than the size of the galaxy, i.e. when

$$
\frac{c}{\nu_{\text {giro }}}=\frac{m c^{2}}{e B}=\frac{E}{e B}=L
$$

( $e$ is the charge of the cosmic proton, $E$ its energy, and $L$ the "size" of the galaxy, for this purpose about twice the width of the disc, say $1 \mathrm{kpc}$ ). If $B$ is taken to be as $5 \times 10^{-6} \mu \mathrm{G}$, energies below $E=e B L \approx 5 \times 10^{9}$ Gev will correspond, in general, to bounded chaotic orbits, isotropically detected at the Earth.

We could use a similar argument to obtain a very rough estimate of the extragalactic magnetic field. Now, we find that energies above $10^{10} \mathrm{Gev}$ correspond to extragalactic cosmic rays. Their arrival is far from isotropic, but let us ignore this important fact, take $L \approx 10 \mathrm{Mpc}$ and obtain $1 \mathrm{nG}$ for the extragalactic field.

Ly- $\alpha$ forests or other pre-galactic structures, already had $\mu \mathrm{G}$ fields. The dynamo is then necessary to order magnetic lines and achieve a coherent distribution at the galactic scale, and it is necessary as well to avoid dissipation of the field as a result of the turbulent magnetic diffusion, but not for amplifying the strength. See Brandenburg \& Subramanian 2005 as an extensive analysis of dynamos. The pregalactic field should be only three orders of magnitude lower, i.e. $5 \times 10^{-9} \mathrm{G}$ to account for the galactic relative collapse. This is approximately the field that magnetogenesis must obtain and the strength we are looking for in the early universe. In order to compare the field strength $B$ at any cosmic scale factor, $a$, with the present values of the strength, it is customary to define the "equivalent-to-present" or "comoving" strength, $B_{0}$ as

$$
B_{0}=B a^{2}
$$

in order to take into account the expansion effect; therefore, $B_{0}$ is not the field strength today; it would be the present strength if no variation of the field other than that induced by expansion would take place since $z$.

\section{Conductivity}

In all epochs of the Universe, the conductivity can be assumed to be infinite and the field can be neither created nor eliminated, just amplified and ordered, therefore 
the diffusion time becomes very large, larger than the Universe lifetime. This is only a simplified picture. There is an additional diffusion enhanced by turbulence, and a battery, of the type of the Bierman battery, can create small fields without a seed. But the question is if the pre-galactic universe had a high conductivity, so that we are able to envisage fields at very early times.

Consider for instance the post-Recombination epoch, the most resistive one, because the number density of electrons was evidently very low. There are however residual electrons that survived Recombination. Kolb \& Turner (1990) estimated the density of free electrons as about $10^{-10}(1+z)^{3} \mathrm{~cm}^{-3}$, assuming that these free electrons had no opportunity to recombine, once the recombination rate became smaller than the expansion rate. The conductivity is

$$
\sigma=\frac{n_{e}^{2} e^{2} \tau_{T}}{m_{e}}
$$

where $\tau_{T}$ is the time between collisions of the electric chargers which are assumed to be electrons. Collisions are mainly due to CMB photons, through Thomson scattering, therefore

$$
\tau_{T}=\frac{1}{n_{\gamma} \sigma_{T}}
$$

and therefore

$$
\sigma=\frac{n_{e}^{2} e^{2}}{m_{e} n_{\gamma} \sigma_{T}}
$$

Here $n_{\gamma} / n_{e}$ should be proportional to the specific entropy of photons per baryons, which is constant if the universe fluid is ideal. Finally we find $\sigma \approx 10^{11} \mathrm{~s}^{-1}$. Due to the constancy of $n_{\gamma} / n_{e}$ the conductivity is a constant after Decoupling. With this value of the conductivity we can find the maximum size of a magnetic coherence cell that can survive until today. The diffusion time is calculated with

$$
\tau_{\text {diff }}=\frac{4 \pi \sigma L_{B}^{2}}{c^{2}}
$$

$L_{B}$ is the characteristic length of the coherence cell. Substituting $\tau_{\text {diff }}$ by the life-time of the Universe $t_{0}$, we obtain that the characteristic diffusion length is only about $1 \mathrm{AU}$. Therefore, even in the most resistive post-Recombination era, the most adverse for the maintenance of magnetic fields, the assumption of infinite conductivity is very reasonable.

\section{Comments on magnetogenesis}

We must then find a mechanism able to create magnetic fields; i.e. without a seed. In general, these mechanisms should produce a positive-negative charge separation, producing a small electric field with non vanishing curl. The magnetic fields created at any epoch should have the strength and the scale large enough to reach the pre-galactic time satisfying the astrophysical requirements.

The many different magnetogenesis theories can be classified into four groups (e.g. Battaner \& Lesch (2000), Battaner \& Florido (2000) and references therein):

a) After Recombination, b) During the Radiation Dominated era, c) in Cosmological Phase Transitions, d) in Inflation. Let us briefly comment on them:

- After Recombination.

Rees (2005) has proposed that the field could be generated rather recently in material ejected by a first generation of supernovae or by population III stars. Intergalactic magnetic fields can be the result outflows from starburst galaxies, like M82 (Kronberg 2005). A generation of M82-type galaxies could inject important quantities of magnetic fields 
into the cluster medium, as it is directly appreciated (Reuter, Klein, Lesch et al. 1994). Massive black holes in the center of galaxies have also been considered by Kronberg as seeding intergalactic fields too.

Therefore, the possibility of a very recent origin of the intergalactic fields from galactic and stellar ejections is very appealing. It does not exclude, however, other ultra-early magnetogenetic processes, as these recent mechanisms do not forcedly rule out others.

- During the Radiation Dominated era.

Harrison (1973) considered a relation between vorticity and magnetic field. The Jeans mass after annihilation is very small, rendering turbulence at the beginning of this era rather peculiar.

- In cosmological phase transitions. Hogan (1983) early proposed cosmological phase transitions as a source of primordial magnetic fields. The electroweak phase transition (at $10^{16} \mathrm{~K}=100 \mathrm{GeV}$ at $a=10^{-16}$, approximately at $10^{-12} s$, being the Hubble radius about $3 \mathrm{~cm}$, have considered by many authors (see the review by Enqvist 1998). The QCD phase transition (at $3 \times 10^{12} \mathrm{~K}=100 \mathrm{MeV}$ at $a=10^{-12}$ with a Hubble radius of $4 \times 10^{4} \mathrm{~m}$ ) has also been extensively studied (e.g. Cheng \& Olinto 1994). Even GUT phase transition has been proposed (e.g. Brandenberger, Davis, Matheson et al. 1992). In general, phase transitions produce large enough strengths but very small lengths of the coherence cells. A very general argument can illustrate this fact.

If the mechanism was completely efficient at the ith phase transition to provide the maximum energy density, then:

$$
\frac{B_{i}}{8 \pi}=a_{r} T_{i}^{4}
$$

where $a_{r}$ is the radiation constant. The real magnetic strength should be less than the value obtained with this equipartition. Then:

$$
B_{0 i}=B_{i} a_{i}^{2}=\left(8 \pi a_{r}\right)^{1 / 2} T_{i}^{2} a_{i}^{2}=\left(8 \pi a_{i}\right)^{1 / 2} T_{0}^{2} \approx 4 \times 10^{-6} G
$$

therefore and rather interestingly, independent of $i$, that is, independent of the phase transition considered. There is a compensation: the earlier the phase transition, the higher the temperature, but the higher the dilution of the field by expansion. The value of the equivalent to present field strength $B_{0 i}$ is higher than requested, but we cannot expect that the mechanism was so efficient.

Concerning the coherence cell size, however, the results is worst. As mechanisms based on phase transitions are causal, the maximum length would correspond to the horizon at that epoch. Before Recombination the Universe expanded as $t^{1 / 2}$, therefore, we have for this maximum size

$$
\lambda_{i}=c t_{i}=c\left(a_{i} / a_{R}\right)^{2} t_{R}=c\left(T_{R} / T_{i}\right)^{2} t_{R}=\left(c T_{R}^{2} t_{R}\right) T_{i}^{-2}
$$

where the subindex $R$ means Recombination. After expansion:

$$
\lambda_{0 i}=\lambda_{i} / a_{i}=\lambda_{i} T_{i} / T_{0}=\left(c T_{R}^{2} t_{R} / T_{0}\right) T_{i}^{-1} \approx 10^{30} T_{i}^{-1}
$$

For the electroweak phase transition with $T_{i}=10^{16} \mathrm{~K}$ we obtain $\lambda_{0 i}=10^{16} \mathrm{~cm}=$ $3 \times 10^{-3} p c$ which is very small for cosmic lengths of interest. In general, the correlation length will be much less than this. For the QCD transition, with a temperature three orders of magnitude lower, the scale is higher but, in any case, insufficient.

This problem could be, in part, alleviated if an inverse cascade transfers the magnetic energy from lower turbulence scales to larger. This inverse cascade has been applied and numerically reproduced in other astrophysical systems (see, for instance, Brandenburg \& Subramanian 2005). The inverse cascade effect is more efficient when the magnetic field has helicity. Helicity is a topological quantity which is conserved along the history of the 
Universe. Some magnetogenesis process have been proposed producing helical magnetic fields (see Kahniashvili (2005), for references). Giovannini 2006 estimate that even with inverse cascade in helical fields the present coherence cells are too small $(<100 \mathrm{pc})$.

- In Inflation. This possibility is extremely attractive. It can give fields at any scale in the same way that super-horizon energy density structures are created and observed. Turner \& Widrow (1988) first proposed an Inflation scenario for the creation of primordial magnetic fields. The idea is exactly the same to explain CMB anisotropies on angular scales larger than the horizon. A coherence cell with present size $\lambda$ has had at any epoch a size of $a \lambda$. This must be compared with the horizon that is a varying function of $a$, During the first phase of Inflation the horizon is independent of $a$, varies as $a^{3 / 2}$ during reheating, as $a^{2}$ along the Radiation era, as $a^{3 / 2}$ along the Matter dominated era. The cell could be sub-horizon when it was created, become super-horizon as a result of the inflationary exponential expansion, and sub-horizon again at photon decoupling. As an example, quantum-mechanical fluctuations could produce electromagnetic waves. The oscillating electric and magnetic fields will appear as static fields when the fast expansion catch up with the wavelength. In later eras electric fields will be canceled by the high conductivity. Gasperini, Giovannini \& Veneziano 1995 first considered the potential relation between inflation fields and CMB.

Very promising is the supersymmetric theory and pre-Big-Bang type models (Gasperini, Giovannini \& Veneziano 1995). The reader is addressed to excellent recent reviews (Giovannini 2002, Giovannini 2006). Supersymmetric theories are able to provide field strengths as high as $10^{-8} \mathrm{G}$ and scales any length, including Sachs-Wolfe scales, only limited in the very small ones by diffusion and Silk damping. This scenario can even provide the initial spectrum of primordial fields (Giovannini 2006).

Though considering the exoticism of theories in the ultra-early Universe, the inflationary magnetogenesis may satisfy the astrophysical requirements. However, Kahniashvili, Maravin \& Kosowsky (2008) have deduced an upper limit of $0.7 \mu \mathrm{G}$ for scales of $100 \mathrm{Mpc}$.

\section{The large scale structure}

The paradigm $\Lambda$ CDM gives a very good overall description of the evolution of large scale structures until the formation of galaxies, clusters and superclusters, but some failures are to be considered, which suggest that the physics should be enriched, or even substantially modified. Probably, magnetic fields are not ignorable and may solve some of these failures. The new data about very large magnetic fields ( $84 \mu G$ in a pregalactic structure at $z \approx 0.7$, Wolfe, Jorgenson, Rodishwa et al. (2008) and those of Kronberg, Bernet, Miniati et al. 2008 prevent us about a premature ignorance of magnetic effects).

Some failures or unexpected results are well known, in particular concerning galaxy formation. The rotation curve is unsatisfactorily explained (Navarro \& Steinmetz 2000), being the simulated Tully-Fisher relation far from the observational one by more than an order of magnitude, the simulated distribution of galactic DM do not well fit the observed rotation curves (de Block, Bosma \& McGaugh 2003). Probably, rotation curves of spiral galaxies, with their important cosmological implications, cannot be understood without the inclusion of magnetism in the dynamics (Battaner and Florido 1992, 1995, 2000, 2007).

The influence of magnetism may be non negligible since very early times, thus not only affecting the birth and structure of galaxies and clusters but the large structure itself (e.g. Wasserman 1978; Giovannini 2004). The variations in the barotropic in$\operatorname{dex}(w$ in the so called equation of state) has an important influence on the metric perturbations (Giovannini 2007). Battaner, Florido \& Garcia-Ruiz (1997), Battaner, 
Florido \& Jiménez-Vicente, J. (1997), Battaner (1998), Florido \& Battaner (1997), and Battaner \& Florido (2000) have considered the effects of magnetic fields in the radiation dominated era strengths in the range $10^{-9}-10^{-8} \mathrm{G}$. The magnetic field has its own barotropic index equal to $1 / 3$. Fields larger than these excessively accelerate galaxy formation, and lower than these, render their effects unimportant. Along this epoch a linear perturbation analysis in the RG Maxwell, fluid and Einstein Field equations is perfectly justified.

Primordial magnetic fields originated at Inflation could acquire a filamentary structure at a large scale. These filaments could connect to form loops that could be the siege of present large scale filaments and voids. Magnetic fields affect the motion of the tightly coupled photon-electron-baryon fluid not only through the Lorentz force, but mainly because they must be included in the energy-momentum tensor, thus introducing an anisotropic gravitating effect. This gravitating magnetic field would produce radiation filaments along this era, DM will fall in these potential wells and after decoupling magnetic fields-DM-baryon filaments would remain. Well after decoupling, non-linear effects would tangle the fields, increasing the strength by about three orders of magnitude, rendering the early distribution nearly impossible to detect today. Superclusters, clusters and galaxies could then be formed out of relic magnetic flux tubes, being distributed along fossil interconnected filaments, which are today observed. It is to be emphasized that gravity alone is able to produce filaments too. Pure gravitational driven filaments cannot possess scales larger than about $50 \mathrm{Mpc}$.

WMAP found an unexpected high Re-Ionization redshift (Kogut et al. 2003). It is possible that magnetic fields can have accelerated the ionizing stellar formation responsible of the premature re-ionization. Subramanian (2006) estimated that at $\mathrm{z}>15$ objects with dwarf galaxy masses and smaller could be the results of cosmic $0.1 \mathrm{nG}$ fields.

Small scale effects can also be produced by PMF. At $z=1$, a typical redshift is the Hubble Deep Field, the intergalactic magnetic energy density was larger by a factor $(1+z)^{4} \approx 16$, thus been able to affect galaxy structure, for instance contributing for producing warps (Reshetnikov, Battaner, Combes et al. 2002).

\section{Magnetic fields and CMB}

If the generation of magnetic fields took place before photon decoupling there is the possibility and the hope that they can be observed in CMB. At present no clear signs of magnetic fields in CMB experiments have been found, but there is a interesting expectation in forthcoming data.

Before collapsing the field was $10^{-10}-10^{-8} \mathrm{G}$. Hence $\Omega_{B}$ is about $10^{-5} \Omega_{\gamma}$. As both energies vary as $a^{-4}$, this relation should hold at Decoupling. As $10^{-5}$ is the level of observed CMB anisotropies, there is the reasonable hope that the cosmic magnetic field has left observable traces in the CMB.

The physics is more exotic when compared with terrestrial standards, but not so much. The magnetic field strength is, as we have seen, of the order of $10^{-8}(z+1)^{2}$ about $10^{-2}$ $\mathrm{G}$ which is close to a typical value in the terrestrial high atmosphere. The density at Decoupling is of the order of the critical density times $(1+z)^{3}$ about $10^{-20} \mathrm{gr} \mathrm{cm}^{-3}$, also very similar to the atmospheric density at, say, $1000 \mathrm{~km}$ high and the temperature at $z \sim 1000$ was also similar to the upper thermosphere. It is to be noticed that the first detections of the CMB were obtained (though not with this purpose) in the high atmosphere. To emphasize even more the familiarity of the CMB medium, note that the anisotropy power spectrum, with the main Doppler peak and other smaller peaks reminds more music than noise, and given the fact that the anisotropies are found with $\mu K$ over temperatures of $\sim 2.7 K$, we could measure the amplitude of this music corresponding to few decibels, i.e. a barely perceptible music. 
These coincidences being noticeable, the physics in the last scattering surface and in the terrestrial upper thermosphere also present important differences, such as the Hubble expansion, the initial conditions corresponding to the radiation dominated era and earlier, the influence of dark matter and energy, the collapse of inhomogeneities, and other facts that render our knowledge of the terrestrial atmosphere of little help for studying CMB.

The most direct measure of magnetic fields present in the last scattering surface would be achieved by direct detection of Faraday Rotation (FR), which is very difficult, but the hope is not completely lost. In order to estimate the order of magnitude of the rotation angle can be obtained by an approximated argument (Kosowsky \& Loeb 1997):

In our case the source and the FR medium is the same. The polarization mechanism is Thomson scattering (under certain quadrupole distribution of the electron density) and the optical depth is:

$$
\tau_{T}=\int \sigma_{T} n_{e} d l
$$

What are the limits of the integral, i.e. how deep is the source and the transmission medium?. It cannot be $\Delta z$, the width of the decoupling transition, because only the closest layers within $\Delta z$ drive out polarized light. For an oder of magnitude we could set $\tau_{T}=1$, hence:

$$
\int n_{e} d l=\frac{1}{\sigma_{T}}
$$

For the rotation angle we would then get:

$$
\varphi=\lambda^{2} K \int B_{\|} n_{e} d l=\lambda^{2} K B_{\|} \frac{1}{\sigma_{T}}
$$

But $B_{\|} \lambda^{2}$ is invariant $\left(B \propto a^{-2}, \lambda \propto a\right)$ hence, $B_{\|} \lambda^{2}=\left[B_{\|} \lambda^{2}\right]_{0}$ at present. For 30 $\mathrm{GHz}, \lambda=1 \mathrm{~cm}$ if $B \approx=10^{-8} \mathrm{G}$ we obtain $\varphi=0.36 \mathrm{rad}=20^{\circ}$. Kosowsky \& Loeb 1997, Kosowsky \& Loeb (2005) carried out a detailed calculation and obtained $\varphi=1$ for $B=10^{-9} \mathrm{G}$. An angle of 20 degrees is perfectly measurable by Planck. However the main difficulty is that the signal is too noisy, as the polarization intensity is very low. Another important difficulty is that we need to observe the primordial Faraday Rotation across the Milky Way. A very precise model of the galactic magnetic field distribution at the galactic large scale is required in order to find clean windows for observing primordial $\mathrm{FR}$, and to decontaminate the galactic contribution.

However, FR could affect the polarization spectra, identifiable as due to magnetic field driven perturbations because of its $\lambda^{2}$-dependence. In particular, FR can convert E polarization modes into $B$ modes $(E$ and $B$ are polarization modes, similar to the Stokes parameters but rotational invariants) and could give appreciable traces into the polarization power spectra. The polarization in the $E$ and $B$ modes has been calculated by Scoccola, Harari \& Mollerach (2004) and Lewis (2004). Kosowsky \& Loeb (2005) and Kosowsky \& Loeb (2005) have detaily calculated this effect by considering stochastic magnetic fields. FR should produce low power polarization spectra, unless frequencies lower than $30 \mathrm{GHz}$ and arcmin angular resolutions are used. These values renders the detection of BMF $\mathrm{FR}$ in $E$ and $B$ polarization spectra rather challenging. Helical fields produce no FR at all. Faraday Rotation can also have a net depolarization effect (Harari, Hayward \& Zaldarriaga 1997).

To reproduce the effect of magnetic fields, mainly on the angular power spectrum a rather large list of possible choices complicate the models. Initial conditions may be of isocurvature or of adiabatic type. The initial spectrum may be assumed to be a power low or not. Some authors adopt a mean field homogeneous, other completely inhomogeneous, 
i.e. stochastic magnetic fields, or any other intermediate arrangement. The field may be helical or not. The perturbations induced in the metric may be scalar, vector or tensor modes, being magnetic fields able to produce the three types. The gauge may be synchronous, longitudinal or others, etc. To account for so many choices would render this review too extended.

Some works by Caprini \& Durrer (2001), and Caprini \& Durrer (2006) have shown that the continuous production of gravity waves, render magnetic strengths very low at Recombination. If primordial magnetic fields are produced by a causal process, as the electroweak phase transitions is, they estimate that the strength should be less than $10^{-27} \mathrm{G}$ and even lower if they are produced at Inflation. Only large scales would be free of this constraint for invariant scale produced fields close to -3 , being $n+3$ the exponent of the assumed power low spectrum of primordial fields. A controversy (Kosowsky \& Loeb (2005); Caprini \& Durrer 2005) have followed this work.

Durrer, Kahniashvili \& Yates (1998) have shown that in an homogeneous magnetic field, Alfvèn waves should produce a testable correlation between different coefficients of the spherical harmonic analysis, in particular

$$
D_{l(m)}=<a_{l-1, m} a_{l+1, m}^{*}>\neq 0
$$

which should vanish under perfect Gaussian conditions. This would constitute a testable prediction with no other interpretation than magnetic fields, indicating a preferred direction in the Universe. This would hold for any multipol scale lower than Silk damping scale at about $l=500$.

There are some recent codes that are an important tool to analyze the forthcoming Planck data considering the effects of magnetic fields. In a series of papers Giovannini and Kunze have contributed with a valuable work to understand Planck results. Giovannini 2007 first developed a semianalitical model for the temperature spectrum, for a nearly scale invariant fully inhomogeneous primordial field, covering the Sachs-Wolfe zone $(1<30)$ and the Doppler zone $(1<100)$. This is an efficient strategy to analytically try to obtain results before dealing with numerical computations. This semianlytical model was followed by a modification of the popular CMBFAST to include magnetic fields (Giovannini and Kunze, 2008 a,b,c,d,e) in which they estimated the anisotropy temperature and polarization spectra simulated maps and the Faraday Rotation. The first and third Doppler peaks increase, the second is distorted and the field increases the number of both hot and cold peaks, among other reliable results.

The non magnetic parameters were kept the same as in the so called concordance model. Relatively large magnetic strengths are needed to modify the standard interpretation, but measurable in most cases. The authors found possible remarkable and warning degeneracies. For example, a lower DM could be compensated with magnetic fields.

Clearly, a blind simultaneous estimation of the cosmological parameters - the standard ones plus two magnetic- would be the final aim of this research about what these authors designate as the $\mathrm{m} \Lambda \mathrm{CDM}$, where $m$ states for magnetic.

Finelli, Paci \& Paoletti (2008) and Paoletti, Finelli \& Paci (2008) have developed another code to study scalar, vector and tensor perturbations from stochastic primordial magnetic fields and their impact on the temperature and polarization spectra, which will become a useful tool to understand the forthcoming Planck maps. The scalar modes are more important for low multipols and the vector modes dominate at large multipols. Magnetic fields are another potential source of non-gaussianity (e.g. Brown \& Crittenden (2005), Brown (2008), Naselsky, Chiang, Obsen et al. 2004). 


\section{Concluding thoughts}

The possibility of a magnetized Universe is not new. We have quoted the early works by Fermi (1949). Even so, this is not the first idea. This merit corresponds to Lemaitre in 1933, as quoted by Peebles (1993). Lemaitre suggested that magnetic flux was conserved in the bounces of the oscillating universe, so that the last generation of galaxies before a bounce were able to became the nucleation of the next generation after the bounce, thus establishing a naive connection between the unending turns of the cycloidic Universe.

Many years before, Faraday, looking for an unification of light, electricity, magnetism and gravity, put a powerful electro-magnet in the path of a plane polarized light ray and observed the today called Faraday Rotation, establishing the basis of the most available tools to measure $B$ at large $z^{\prime} s$.

It has been demonstrated that magnetic fields are dynamically important in a large variety of astrophysical systems. May be, in months, we can complete the list by measuring magnetic fields at $\mathrm{z}=1000$, even witness of much earlier fields. The difficulties being recognized, the $\mathrm{m} \Lambda \mathrm{CDM}$ is in progress. As stated by Peebles (1993): If magnetic fields existed before galaxies, it will be a fascinating hint to what happened in the early universe and a considerable challenge to conventional ideas.

\section{Acknowledgements}

Projects: FQM108, FQM792, AYA2004-08251-C02-02, AYA2007-67625-C02-02.

\section{References}

Battaner, E. 1998, A\&A 334, 770

Battaner, E. \& Florido, E. 1995, MNRAS 277, 1129

Battaner, E. \& Florido, E. 2000, Fund. Cosmic Phys. 21, 1

Battaner, E. \& Florido, E. 2007, AN 328, 92

Battaner, E., Florido, E., \& Garia-Ruiz, J. M. 1997, A\&A 327, 8

Battaner, E., Florido, R., \& Jiménez-Vicente, J. 1997, A\&A 326, 13

Battaner, E., Garrido, J. L., Membrado, M., \& Florido, E. 1992, Nature 360, 652

Battaner, E. \& Lesch, H. 2000, Anal. de Fisica 95, 2000 (astro-ph/0003370)

Battaner, E. \& Rubiño-Martin, J. A. 2008, Constraints on primordial magnetic fields. Project Satus, Planck Working Group 4.

Beck, R. 2005, in: R. Wielebinski \& R. Beck (eds.) in Cosmic Magnetic Fields. Lecture Notes in Physics (Heidelberg: Springer), vol. 664

Beck, R. 2002, in: A. Esquivel et al. (eds.) Magnetic Fields in the Universe II Rev. Mex. Astron. Astrof. (arXiv 0804.4594)

Bernet, M. L., Miniati, F., Lilly, S. J., Kronberg, P. P., \& Dessauges-Zavadski, M. 2008, Nature 454,302

de Block, W. J. G., Bosma, A., \& Mc Gaugh, S. 2003, MNRAS 340, 657

Bosma, A. 2004, in: S. D. Ryder, D. J. Pisano, M. A. Walker \& K. C. Freeman (eds.), Astron. Soc. Pacific, IAU Symp. 220

Brandenberger, R. H., Davis, A. C., \& Matheson, A. M. et al. 1992, Phys. Lett. B 293,287

Brandenburg, A. 2005, in: R. Wielebinski \& R. Beck (eds.) in Cosmic Magnetic Fields. Lecture Notes in Physics (Heidelberg: Springer), vol. 664

Brandenburg, A. \& Subramanian, K. 2005, Phys. Rep. 417, 1

Brown, I. A. 2008, Ph.D. University of Portsmouth

Brown, I. \& Crittenden, R. 2005, Phys. Rev. D 72, 063002

Caprini, C. \& Durrer, R. 2001, Phys. Rev. D 65, 023517

Caprini, C.\& Durrer, R. 2005, Phys. Rev. D 72, 088301

Caprini, C. \& Durrer, R. 2006, Phys. Rev. D 74, 321

Cheng, B. \& Olinto, A. 1994, Phys. Rev. D 50, 2421

Durrer, R., Kahniashvili, T., \& Yates, A. 1998, Phys. Rev. D 58, 123004 
Enqvist, K. 1998, Int. J. Mod. Phys. D 7, 331

Finelli, F., Paci, F., \& Paoletti, D. 2008, Phys. Rev. D 78, 023510

Florido, E. \& Battaner, E. 1997, A\& A 327, 1

Gasperini, M., Giovannini, M., \& Veneziano, G. 1995, Phys. Rev. D 52, 6651

Giovannini, M. 2002, hep-ph/0208152

Giovannini, M. 2004, Int. J. Modern Phys. D 13, 391

Giovannini, M. 2006, astro-ph/12378

Giovannini, M. 2007, Phys. Rev. D 76, 103508

Giovannini, M. \& Kunze, E. 2008a, Phys. Rev. D 77, 063003

Giovannini, M. \& Kunze, E. 2008b, Phys. Rev. D 77, 061301

Giovannini, M. \& Kunze, E. 2008c, arXiv 0804.2238

Giovannini, M. \& Kunze, E. 2008d, Phys. Rev. D 77, 123001

Giovannini, M. \& Kunze, E. 2008e, Phys. Rev. D 78, 023010

Grasso, D. \& Rubinstein, H. R. 2001, Phys. Rep. 348, 163

Harari, D. D., Hayward, J. D., \& Zaldarriaga, M. 1997, Phys. Rev. D 55, 1841

Harrison, E. H. 1973, MNRAS 165, 185

Hogan, C. J. 1983, Phys. Rev. Lett. 51, 1488

Kahniashvili, T. 2005, New Astron. 49, 79

Kahniashvili, T., Maravin, Y., \& Kosowsky, A. 2008, arXiv 0806.1876

Kogut, A. et al. 2003, ApJ 665, 355

Kolb, E. W. \& Turner, M. S. 1990, The early Universe. Adison-Wesley

Kosowsky, A., Kahniashvili, T., Lavrelashvili, G., \& Ratra, B. 2005, Phys. Rev. D 71, 043006

Kosowsky, A. \& Loeb, A. 1997, ApJ 469, 1

Kosowsky, A. \& Loeb, A. 2005, Phys. Rev. D 70, 043011

Kronberg, P. P. 2005, in: R. Wielebinski \& R. Beck (eds.) in Cosmic Magnetic Fields. Lecture Notes in Physics (Heidelberg: Springer), vol. 664, p. 9

Kronberg, P. P., Bernet, M. L., Miniati, F., Lilly, S., Short, M. B., \& Higdon, D. M. 2008, ApJ 676,70

Lewis, A. 2004, Phys. Rev. D 70, 043518

Naselsky, P., Chiang, L. Y., Obsen, P., \& Verkhodanov, O. V. 2004, ApJ 615, 45

Navarro, J. \& Steinmetz, M. 2000, ApJ 528, 607

Paoletti, D., Finelli, F., \& Paci, F. 2008, astrp-ph/0811.0230

Peebles, P. J. E. 1993, Principles of Physical Cosmology, Princeton Series in Physics

Rees, M. 2005, in: R. Wielebinski \& R. Beck (eds.) in Cosmic Magnetic Fields. Lecture Notes in Physics (Heidelberg: Springer), vol. 664

Reshetnikov, V., Battaner, E., Combes, F., \& Jiménez-Vicente, J. 2002, A $\& A$ A 382, 513

Reuter, H. P., Klein, U., Lesch, H., Wielebinski, R., \& Kronberg, P. P. 1994, A\& A 282, 724

Rubiño-Martín, J. A. et al. 2008, arXiv 0810.3141

Scoccola, C., Harari, D., \& Mollerach, S. 2004, Phys. Rev. D 70, 063003

Subramanian, K. 2006, AN 327, 403

Turner, M. S. \& Widrow, L. M. 1988, Phys. Rev. D 37, 2743

Wasserman, I. 1978, ApJ 224, 337

Widrow, L. M. 2002, Rev. Mod. Phys. 74, 775

Wolfe, A. M., Jorgenson, R. A., Robishaw, T., Heiles, C., \& Prochaska, J. X. 2008, Nature 455, 638

\section{Discussion}

DuRRER: Might it not be that just the magnetic field spectrum is too blue to observe them on the large scales probed by CMB experiments like Planck?

BATTANER: I agree, considering the limits you have commented on in your talk. But theory is still poorly supported by observations. I think that Planck remains an interesting possibility to observe PMF (or to establish precise observational bounds). Let's wait for Planck. 